\title{
RESEARCH
}

Open Access

\section{The interplay between metabolic alterations, diastolic strain rate and exercise capacity in mild heart failure with preserved ejection fraction: a cardiovascular magnetic resonance study}

Masliza Mahmod ${ }^{1,2^{*}}$ (D, Nikhil Pal ${ }^{3}$, Jennifer Rayner', Cameron Holloway', Betty Raman', Sairia Dass', Eylem Levelt', Rina Ariga', Vanessa Ferreira', Rajarshi Banerjee ${ }^{4}$, Jurgen E. Schneider ${ }^{1}$, Christopher Rodgers ${ }^{5,6}$, Jane M. Francis ${ }^{1}$, Theodoros D. Karamitsos ${ }^{1,4}$, Michael Frenneaux ${ }^{7}$, Houman Ashrafian ${ }^{3}$, Stefan Neubauer ${ }^{1}$ and Oliver Rider ${ }^{1}$

\begin{abstract}
Background: Heart failure (HF) is characterized by altered myocardial substrate metabolism which can lead to myocardial triglyceride accumulation (steatosis) and lipotoxicity. However its role in mild HF with preserved ejection fraction (HFpEF) is uncertain. We measured myocardial triglyceride content (MTG) in HFpEF and assessed its relationships with diastolic function and exercise capacity.

Methods: Twenty seven HFpEF (clinical features of HF, left ventricular EF $>50 \%$, evidence of mild diastolic dysfunction and evidence of exercise limitation as assessed by cardiopulmonary exercise test) and 14 controls underwent ${ }^{1} \mathrm{H}$-cardiovascular magnetic resonance spectroscopy ( ${ }^{1} \mathrm{H}$-CMRS) to measure MTG (lipid/water, \%), ${ }^{31} \mathrm{P}$-CMRS to measure myocardial energetics (phosphocreatine-to-adenosine triphosphate - PCr/ATP) and feature-tracking cardiovascular magnetic resonance (CMR) imaging for diastolic strain rate.

Results: When compared to controls, HFpEF had 2.3 fold higher in MTG $(1.45 \pm 0.25 \%$ vs. $0.64 \pm 0.16 \%, p=0.009)$ and reduced PCr/ATP (1.60 \pm 0.09 vs. $2.00 \pm 0.10, p=0.005)$. HFpEF had significantly reduced diastolic strain rate and maximal oxygen consumption $\left(\mathrm{VO}_{2}\right.$ max), which both correlated significantly with elevated MTG and reduced PCr/ATP. On multivariate analyses, MTG was independently associated with diastolic strain rate while diastolic strain rate was independently associated with $\mathrm{VO}_{2}$ max.

Conclusions: Myocardial steatosis is pronounced in mild HFpEF, and is independently associated with impaired diastolic strain rate which is itself related to exercise capacity. Steatosis may adversely affect exercise capacity by indirect effect occurring via impairment in diastolic function. As such, myocardial triglyceride may become a potential therapeutic target to treat the increasing number of patients with HFpEF.
\end{abstract}

Keywords: Cardiovascular magnetic resonance, Spectroscopy, Diastolic strain rate, Heart failure, Steatosis, Maximal oxygen consumption

\footnotetext{
* Correspondence: masliza.mahmod@cardiov.ox.ac.uk

'Division of Cardiovascular Medicine, Radcliffe Department of Medicine, University of Oxford Centre for Clinical Magnetic Resonance Research (OCMR), University of Oxford, John Radcliffe Hospital, Headley Way, Oxford OX3 9DU, UK

${ }^{2}$ National University of Malaysia Medical Centre, Kuala Lumpur, Malaysia

Full list of author information is available at the end of the article
}

(c) The Author(s). 2018 Open Access This article is distributed under the terms of the Creative Commons Attribution 4.0 International License (http://creativecommons.org/licenses/by/4.0/), which permits unrestricted use, distribution, and reproduction in any medium, provided you give appropriate credit to the original author(s) and the source, provide a link to the Creative Commons license, and indicate if changes were made. The Creative Commons Public Domain Dedication waiver (http://creativecommons.org/publicdomain/zero/1.0/) applies to the data made available in this article, unless otherwise stated. 


\section{Background}

Almost half of all patients who present with clinical features of heart failure (HF) have preserved left ventricular (LV) ejection fraction (HFpEF). Its prevalence is on the rise, representing a major burden for health care services [1]. These patients are often elderly, female with multiple co-morbidities such as hypertension and obesity, and typically show a non-dilated LV, concentric remodelling and abnormal diastolic function [2]. The diagnostic criteria of HFpEF is based on clinical features of HF and normal LV ejection fraction (EF) together with evidence of diastolic dysfunction, LV hypertrophy, left atrial (LA) enlargement and raised plasma brain natriuretic peptides (BNP) according to the current European Society of Cardiology (ESC) guidelines [3].

However, the current criteria focus on patients with more advanced stages of the diseases leaving a large proportion of patients with mild diastolic dysfunction and limiting dyspnoea which may be overlooked in daily clinical practice. In addition, the vast majority (90\%) of elderly patients with isolated moderate/severe diastolic dysfunction and normal LVEF not diagnosed to have HF have limiting dyspnoea [4]. Furthermore, nearly a third of asymptomatic patients with diastolic dysfunction develop symptoms of breathlessness, oedema or fatigue over 2 years [5] and this progression is accompanied by a substantial decrease in survival rate [6]. As a result, there is a real need for novel and, effective therapeutic targets to improve the management of the increasing number of patients with HFpEF.

Myocardial metabolism is a promising therapeutic target in HF [7]. HF is characterized by impaired myocardial energetics and altered myocardial substrate metabolism with a switch in fatty acid (FA) oxidation towards glucose oxidation for adenosine triphosphate (ATP) generation. This metabolic substrate shift may be a consequence of glucose being energetically more efficient (lower oxygen usage) than fatty acids [7, 8]. Accompanying this switch is an imbalance between FA uptake (which continues to be high) and FA oxidative metabolism (which is reduced), leading to intracellular cardiac lipid accumulation. This accumulation provides a source for non-oxidative metabolism to diacylglycerol and ceramide, potentially resulting in lipotoxicity, apoptosis and cardiac dysfunction [8-10].

Proton $\left({ }^{1} \mathrm{H}\right)$ cardiovascular magnetic resonance (CMR) spectroscopy (CMRS) allows the non-invasive measurement of myocardial lipid (triglyceride) content [11], and by using this technique, we and others have demonstrated that cardiac steatosis occurs in several situations that are characterised by diastolic dysfunction; aortic stenosis, metabolic syndrome, obesity and type 2 diabetes mellitus [12-14]. Whilst steatosis [15] and reduced myocardial energetics [16] have both been shown to be present in HFpEF, metabolic characterization assessing both myocardial triglyceride (MTG) and energetics within the same cohort of patients with HFpEF and their relationship with cardiac function and exercise capacity have not previously been explored. The focus of this study was to investigate MTG content and energetics in patients with mild HFpEF and their relationship with cardiac function and exercise capacity.

CMR feature-tracking (CMR-FT) is a technique recently developed that can quantify strain and diastolic strain rate based on standard CMR cine balanced steady state free precession (bSSFP) images independent of additional sequences, with considerably reduced post processing time, and has been shown to have good agreement with CMR tissue tagging [17]. Although CMR tissue tagging is the gold standard for measuring cardiac strain, increased scanning time due to acquisition of additional tagging sequences and processing time limits its routine use [17]. We used multi-parametric cine CMR and multinuclear CMRS $\left({ }^{1} \mathrm{H}\right.$ and $\left.{ }^{31} \mathrm{P}\right)$ to investigate metabolic changes in $\mathrm{HFpEF}$, and their relationships with diastolic function (diastolic strain rate) and exercise capacity, as measured by maximal oxygen consumption $\left(\mathrm{VO}_{2} \max \right)$.

\section{Methods}

\section{Study population}

A group of 27 patients with HFpEF defined by the presence of symptoms or signs of HF, a non-dilated LV with LVEF $\geq 50 \%$ and evidence of abnormal diastolic function on Doppler echocardiography were prospectively enrolled between 2011 and 2018 [18, 19]. Additionally, we performed cardiopulmonary exercise testing (CPET) to confirm their exercise limitation by measuring $\mathrm{VO}_{2} \max$ $\left(\mathrm{VO}_{2}\right.$ at peak exercise). Patients were enrolled if their $\mathrm{VO}_{2}$ max was $<80 \%$ predicted for age and gender, with a pattern of gas exchange that would indicate a cardiac cause of exercise limitation. Patients who had diabetes mellitus, uncontrolled hypertension, significant valvular disease, previous myocardial infarction, coronary revascularization, previous cardiac surgery, contraindications to CMR or estimated glomerular filtration rate $<30 \mathrm{ml} / \mathrm{min}$ were excluded. Fourteen age, gender and body mass index (BMI) matched healthy controls without a history of heart disease, diabetes, hypertension or dyslipidaemia were also recruited. They were identified from the local population by word of mouth and poster advertisements around hospital and university. Controls were included if they were 65 years or older, asymptomatic, not on any medications and had no cardiac abnormalities detected on electrocardiogram (ECG) or CMR. All study participants fasted for at least $6 \mathrm{~h}$ prior to CMR and CMRS and were scanned at around the same time of the day. 


\section{Study protocol}

All study participants underwent resting transthoracic echocardiography, ${ }^{31} \mathrm{P}$-CMRS $(n=20$ for HFpEF and $n=10$ for controls) and ${ }^{1} \mathrm{H}$-CMRS in addition to the standard CMR imaging. All HFpEF patients and a subgroup of healthy controls $(n=9)$ underwent CPET.

\section{${ }^{1} \mathrm{H}$ cardiovascular magnetic resonance spectroscopy}

Myocardial ${ }^{1} \mathrm{H}$ spectra were obtained from the mid interventricular septum as previously described [11]. In short, spectroscopic acquisitions were performed using ECG trigger at end-expiration to minimize motion artefacts. Water-suppressed spectra were acquired to measure cardiac lipid, and spectra without water suppression were acquired and used as an internal standard. Spectroscopic stimulated echo (STEAM) sequence with an echo time (TE) of $10 \mathrm{~ms}$ was used. Five to six water-suppressed scans ( 5 averages each; repetition time (TR) of at least $2 \mathrm{~s}$ ) were acquired in mid-diastole in a series of single breath-holds of about $10 \mathrm{~s}$ each. Next, a water spectrum (3 averages; TR of at least $4 \mathrm{~s}$ ) was acquired in a single breath-hold to use as internal reference. Spectra were analysed using a custom Matlab (Mathworks, Natick, Massachusetts, USA) implementation of AMARES (Advanced Method of Accurate, Robust and Efficient Spectroscopic) and the AMARES algorithm in jMRUI (Java-based Magnetic Resonance User Interface) as previously described [11]. Myocardial triglyceride content was calculated as a percentage relative to water: (signal amplitude of lipid/signal amplitude of water) $\times 100 \%$.

\footnotetext{
${ }^{31} \mathrm{P}$ cardiovascular magnetic resonance spectroscopy

${ }^{31} \mathrm{P}$-CMRS was performed to obtain PCr/ATP ratio from a voxel placed in the mid-ventricular septum, with the subjects lying prone with their heart over the centre of the ${ }^{31} \mathrm{P}$ heart/liver coil in the magnet isocentre as previously described [20]. ${ }^{31} \mathrm{P}$-CMRS post processing analysis was performed using a custom Matlab analysis tool, as previously described [20]. Coil positioning was confirmed or adjusted with the use of proton localization images as previously described. Spectra were acquired using acquisition-weighted 3D chemical shift imaging (CSI) [21], with 10 averages in the centre of K-space. The TR was $720 \mathrm{~ms}$ and the acquisition duration was $8 \min$ [22].
}

\section{Cardiovascular magnetic resonance imaging}

Cine CMR images were acquired for cardiac volume analysis using a $3 \mathrm{~T}$ CMR system (Trio, Siemens Healthineers, Erlangen, Germany) using bSSFP cine imaging as previously described [23]. Analysis of cardiac volumes, function and mass was performed using Argus post-processing software (Siemens Healthineers). The systolic circumferential strain and diastolic strain rate parameters were calculated using feature tracking software Circle, Cardiovascular (cvi42 $2^{\circ}$, Circle Cardiovascular Imaging Calgary, Canada) from the short-axis bSSFP cine images. The epicardial and endocardial borders were traced manually at diastole and the software then tracked the deformation throughout the cardiac cycle generating values for global circumferential strain and circumferential diastolic strain rate. In case of insufficient tracking of the endocardial and epicardial borders, contours were manually corrected and the tracking repeated.

\section{Transthoracic echocardiography}

Transthoracic echocardiography (echo) was performed using a commercial echocardiographic system (iE33, Philips Healthcare, Best, The Netherlands). Measurements of LV diastolic function were performed according to the guidelines [24]. The following diastolic indices were obtained; transmitral early (E) and late (A) diastolic velocities, mitral annular early (e') diastolic velocity, with calculation of $\mathrm{E} / \mathrm{A}$ and $\mathrm{E} / \mathrm{e}^{\prime}$ ratios.

\section{Cardiopulmonary exercise test (CPET)}

Cardiopulmonary exercise testing was undertaken using an upright treadmill or bicycle ergometer protocol with simultaneous respiratory gas analysis after performing a spirometry, as described [16]. An incremental ramp protocol was utilised whereby speed and inclination (for treadmill) or resistance and speed (for bicycle exercise) were gradually increased with continual heart rate, blood pressure and ECG recording. All subjects were exercised to volitional fatigue, with a corresponding adequate respiratory exchange ratio (RER) achieved as a requirement for satisfactory effort defined as RER of $>1$. Maximal oxygen consumption $\left(\mathrm{VO}_{2} \max \right)$ was determined by averaging $\mathrm{VO}_{2}$ measures over $30 \mathrm{~s}$ of peak exercise.

\section{Statistical analysis}

A priori sample size calculation was performed which was based on a change in PCr/ATP ratio. With a power of $80 \%$ and $p<0.05$, a sample size of 18 would be required to identify a difference of 0.39 in PCr/ATP. As the true effect size for MTG was unknown in this population, a post-hoc analysis using MTG was performed which showed that 30 subjects (20 HFpEF and 10 controls) would have a power of $86 \%$. Data are expressed as mean \pm SD for description of study cohorts and mean \pm SE for CMR and CMRS variables. Categorical data are presented as numbers and percentages. Comparisons between the two groups were performed by non-parametric method due to small sample size. The Chi-squared test or Fisher's exact test were used to compare categorical data as appropriate. Bivariate correlations were performed in all subjects using Pearson's 
or Spearman's method as appropriate. Non-normally distributed data were log transformed to construct normal data. Variables with significant correlations with diastolic strain rate and $\mathrm{VO}_{2}$ max were entered into a stepwise multivariate model to determine predictors of diastolic strain rate and $\mathrm{VO}_{2}$ max. A $P$-value $<0.05$ was considered significant. All statistical analyses were performed with SPSS (version 21, International Business Machines, Armonk, New York, USA).

\section{Results}

Description of patient cohort and baseline characteristics Sixty-five patients with chronic breathlessness under follow up in the cardiology clinics were identified and screened in addition to 7 patients who were recruited by posters. Of these, 37 were eligible to enter the study, of which the first 31 consecutive patients took part in the study. Four patients were excluded due to incomplete study protocol. None of the patients had prior HF admissions, prior insults such as chemotherapy or radiation. None of the patients had been diagnosed or suspected to have amyloidosis, and CMR did not show findings suggestive of amyloidosis. Patients with history of angina, previous myocardial infarction or coronary revascularization were excluded. Although coronary angiography was not performed prior to entering the study, none of the patients had a history of angina, and they did not have angina or develop any ischaemic changes during CPET.

Table 1 shows demographic, clinical, echocardiographic, biochemical and $\mathrm{VO}_{2}$ max results for both HFpEF and healthy control groups. Both groups were matched with regards to age, gender, BMI and glucose and blood cholesterols. Frequency matching instead of individual (one-to-one) matching was performed which was based upon proportion of the age and gender for

Table 1 Clinical, echocardiographic and biochemical characteristics

\begin{tabular}{|c|c|c|c|}
\hline & $\operatorname{HFpEF}(n=27)$ & Healthy Controls $(n=14)$ & $P$ value \\
\hline Age (years) & $72 \pm 7$ & $69 \pm 6$ & 0.09 \\
\hline Female, n (\%) & $18(67)$ & $8(57)$ & 0.55 \\
\hline \multicolumn{4}{|l|}{ NYHA class, $n(\%)$} \\
\hline । & 0 & $14(100)$ & $<0.001$ \\
\hline$\|$ & $24(89)$ & 0 & $<0.001$ \\
\hline III & $3(11)$ & 0 & $<0.001$ \\
\hline Body mass index $\left(\mathrm{kg} / \mathrm{m}^{2}\right)$ & $29 \pm 6$ & $26 \pm 5$ & 0.10 \\
\hline Hypertension, n (\%) & $12(44)$ & 0 & 0.04 \\
\hline Atrial fibrillation, $n(\%)$ & 0 & 0 & - \\
\hline Beta blockers, $n(\%)$ & $3(11)$ & 0 & 0.53 \\
\hline ARB/ACE inhibitor, $n(\%)$ & $14(52)$ & 0 & 0.004 \\
\hline Diuretics, n (\%) & $12(44)$ & 0 & 0.005 \\
\hline Statins, $n(\%)$ & $11(41)$ & 0 & 0.02 \\
\hline Systolic BP (mmHg) & $144 \pm 26$ & $132 \pm 4$ & 0.15 \\
\hline Diastolic BP (mmHg) & $81 \pm 12$ & $80 \pm 13$ & 0.86 \\
\hline Heart rate (bpm) & $68 \pm 12$ & $60 \pm 12$ & 0.10 \\
\hline E/A ratio & $0.69 \pm 0.23$ & $0.86 \pm 0.24$ & 0.042 \\
\hline E/e' ratio & $10.87 \pm 2.61$ & $7.47 \pm 2.45$ & $<0.001$ \\
\hline LA size $\left(\mathrm{ml} / \mathrm{m}^{2}\right)$ & $31.1 \pm 15.4$ & $16.9 \pm 6.4$ & 0.003 \\
\hline $\mathrm{VO}_{2} \max (\mathrm{ml} / \mathrm{min} / \mathrm{kg})$ & $17.7 \pm 3.3$ & $27.8 \pm 7.7$ & 0.004 \\
\hline Blood glucose (mmol/L) & $5.4 \pm 0.9$ & $4.8 \pm 0.4$ & 0.07 \\
\hline Free fatty acids (mmol/L) & $0.49 \pm 0.28$ & $0.54 \pm 0.19$ & 0.10 \\
\hline Triglycerides (mmol/L) & $1.26 \pm 0.37$ & $1.13 \pm 0.45$ & 0.44 \\
\hline LDL (mmol/L) & $2.45 \pm 0.71$ & $3.17 \pm 1.05$ & 0.03 \\
\hline $\mathrm{HDL}(\mathrm{mmol} / \mathrm{L})$ & $1.52 \pm 0.41$ & $1.60 \pm 0.41$ & 0.59 \\
\hline BNP (pmol/L) & $15.6 \pm 9.4$ & $6.8 \pm 3.8$ & 0.01 \\
\hline
\end{tabular}

Values are mean \pm SD or percentages

$A C E$ Angiotensin-converting enzyme-inhibitors, $A R B$ Angiotensin-receptor antagonist-II, BNP Brain natriuretic peptide, $H D L$ High-density lipoprotein, $H F p E F$ Heart failure with preserved ejection fraction, $L D L$ Low-density lipoprotein 
both cohorts. It was challenging to achieve equal number of controls to the HFpEF group, as a significant number of healthy elderly subjects had to be excluded due to concomitant medical problems. Although the age (72 vs 69 ) and gender (67 vs $57 \%$ ) were not perfectly matched, the differences are numerically small (3 years for age and $10 \%$ for gender), and statistically they were not significant $(p>0.05)$. All HFpEF patients had (1) signs or symptoms of HF, (2) normal LVEF and LV cavity size, (3) evidence of mild diastolic dysfunction on Doppler echocardiography, and, additionally (4), objective evidence of cardiac cause of exercise limitation on CPET. We decided to use the latter as an additional inclusion criterion, as this provided evidence of a cardiac cause of exercise intolerance in our mild HFpEF population, rather than being attributed to physical deconditioning, commonly seen in healthy elderly $[16,25,26]$, and the predicted values are entirely consistent with a meta-analysis of studies from which reference values were developed [27].

\section{Assessment of left ventricular function and geometry}

Table 2 summarises CMR results for both groups. When compared to controls, HFpEF showed concentric remodeling as indicated by increased LV mass to LV end diastolic volume (EDV) ratio but normal LV mass index. As expected diastolic strain rate in HFpEF was significantly impaired. Despite normal LVEF, peak systolic circumferential strain was significantly impaired in HFpEF, indicating additional subtle contractile dysfunction. None of the HFpEF or controls had a late gadolinium enhancement (LGE) pattern indicating previous myocardial infarction. To assess gender difference in LV functional parameters, analyses were performed comparing LVEDV,
LV end systolic volume (ESV) and LVEF between women and men separately in both groups. There were no significant gender differences in LV volumes and function in both groups (results not shown).

\section{Assessment of cardiac metabolism with ${ }^{1} \mathrm{H}$ - and ${ }^{31} \mathrm{P}$ cardiovascular magnetic resonance spectroscopy} Table 2 also shows the comparison of ${ }^{1} \mathrm{H}$ and ${ }^{31} \mathrm{P}$ CMRS results for all study groups. Compared to controls, HFpEF patients had pronounced steatosis (a 2.3-fold increase) and impaired energetics despite similar age, gender and BMI (Fig. 1).

\section{Myocardial triglyceride, energetics, diastolic strain rate and $\mathrm{VO}_{2}$ max}

Elevated MTG (Fig. 2) and reduced PCr/ATP ratio correlated significantly with impaired diastolic strain rate but no significant correlations with other diastolic indices such as transmitral E/A or E/e'. There were no significant correlations observed between MTG and $\mathrm{PCr}$ / ATP with echo diastolic indices, as the echo variables were not powered to detect these differences. On stepwise multivariate regression analysis, MTG but not $\mathrm{PCr} /$ ATP independently correlated with diastolic strain rate (adjusted $R^{2}=0.48$ ) (Table 3). Increased MTG, reduced $\mathrm{PCr} / \mathrm{ATP}$, and impaired diastolic strain rate (Fig. 2) correlated significantly with reduced $\mathrm{VO}_{2}$ max but on stepwise multivariate analysis only diastolic strain rate was an independent correlate of $\mathrm{VO}_{2} \max$ (adjusted $\mathrm{R}^{2}=$ $0.30)$. As expected both MTG $(r=0.35, p=0.027)$ and diastolic strain rate $(r=-0.48, p=0.001)$ correlated significantly with New York Heart Association (NYHA) class suggesting there may be a relationship between cardiac lipid and exercise tolerance.

Table 2 CMR and CMRS results

\begin{tabular}{llll}
\hline & $\begin{array}{l}\text { HFpEF } \\
(n=27)\end{array}$ & $\begin{array}{l}\text { Healthy Controls } \\
(n=14)\end{array}$ & $P$ value \\
\hline PCr/ATP ratio & $1.60 \pm 0.09$ & $2.00 \pm 0.10$ & 0.005 \\
Cardiac lipid/water (\%) & $1.45 \pm 0.25$ & $0.64 \pm 0.16$ & 0.009 \\
Diastolic strain rate (\%/s) & $84.6 \pm 5.3$ & $110.4 \pm 5.5$ & 0.002 \\
Systolic circumferential strain (\%) & $-19.5 \pm 0.4$ & $-21.8 \pm 0.5$ & 0.002 \\
LV end-diastolic volume $\left(\mathrm{ml} / \mathrm{m}^{2}\right)$ & $62 \pm 2$ & $66 \pm 4$ & 0.20 \\
LV end-systolic volume $\left(\mathrm{ml} / \mathrm{m}^{2}\right)$ & $18 \pm 2$ & $20 \pm 2$ & 0.40 \\
LV stroke volume $(\mathrm{ml})$ & $87 \pm 3$ & $92 \pm 6$ & 0.38 \\
LV ejection fraction (\%) & $72 \pm 1$ & $70 \pm 2$ & 0.48 \\
LV wall thickness $(\mathrm{mm})$ & $13 \pm 1$ & $9 \pm 1$ & $<0.001$ \\
LV mass $(\mathrm{g})$ & $101 \pm 5$ & $90 \pm 6$ & 0.22 \\
LV mass index $\left(\mathrm{g} / \mathrm{m}^{2}\right)$ & $54 \pm 3$ & $50 \pm 3$ & 0.39 \\
LV mass/EDV $(\mathrm{g} / \mathrm{mL})$ & $0.86 \pm 0.05$ & $0.68 \pm 0.05$ & 0.03 \\
\hline Vales are meas & & & \\
\hline
\end{tabular}

Values are mean $\pm \mathrm{SE}$

EDV End-diastolic volume, LV Left ventricular, $P C r$ Phosphocreatine, ATP Adenosine triphosphate 


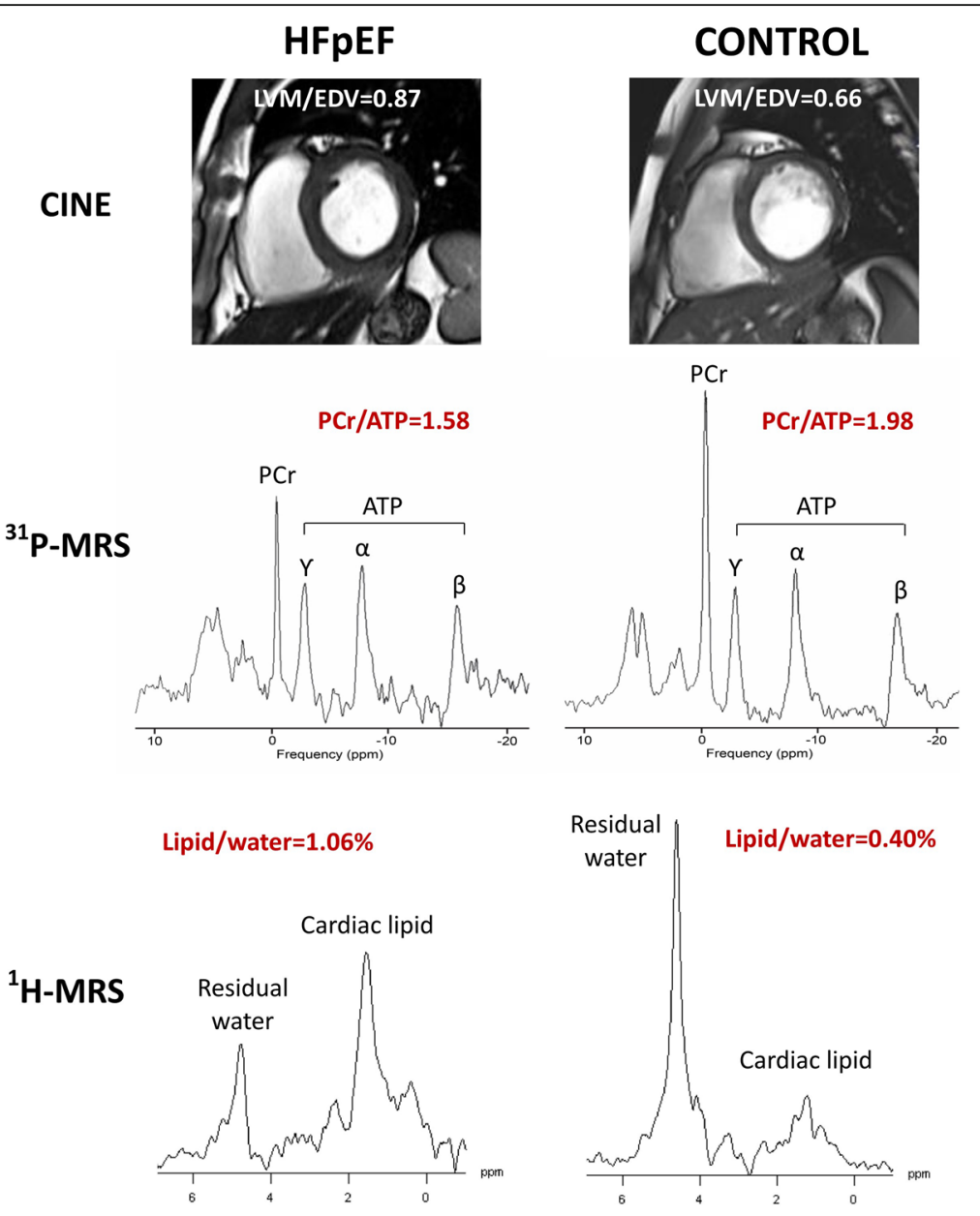

Fig. 1 Cine imaging (top panel), ${ }^{31} \mathrm{P}-\mathrm{CMRS}$ (middle panel) and ${ }^{1} \mathrm{H}-\mathrm{CMRS}$ (bottom panel) showing representative results of LVM/EDV, PCr/ATP and $\mathrm{Lipid} /$ water for heart failure with preserved ejection fraction (HFpEF) (left) and control (right). ${ }^{1} \mathrm{H}$-CMRS spectra are scaled based on unsuppressed water (not shown) and noise level. LVM = left ventricular mass; EDV = end-diastolic volume; CMRS = cardiovascular magnetic

resonance spectroscopy

By using the cut off values of abnormal lipid/water $>$ 0.64 and abnormal PCr/ATP of $<2.0$ from the current study, of the 27 HFpEF patients, $21 / 27$ (78\%) had abnormal lipid/water and 17/20 (85\%) had abnormal $\mathrm{PCr} /$ ATP. Of the 14 controls, 3/14 (21\%) had abnormal lipid and 4/10 (40\%) had abnormal PCr/ATP and the rates of these abnormalities between HFpEF and controls were statistically significant $(p<0.05)$. Furthermore, the patients with abnormal lipid/water and PCr/ATP ratio had significantly lower $\mathrm{LV}$ diastolic strain rate and $\mathrm{VO}_{2}$ max than those with normal lipid/water and $\mathrm{PCr} / \mathrm{ATP}$ ratio (results not shown). By using the cut-off values of abnormal lipid/water $>0.77$ and abnormal PCr/ATP $<1.6$, of the 27 HFpEF patients, 19/27 (70\%) had abnormal lipid/ water and 15/20 (75\%) had abnormal PCr/ATP.

In line with our previous work [13], we found that MTG had a significant correlation with peak systolic circumferential strain $(r=0.61, p<0.001)$. In addition we found that reduced PCr/ATP correlated with impaired peak systolic circumferential strain $(r=-0.55, p=0.002)$ but no significant correlations with age, BMI, systolic blood pressure (SBP) or BNP.

\section{Discussion}

The present study has three major findings. First, there is pronounced myocardial steatosis in patients with mild HFpEF, with a 2.3-fold increase in MTG compared to age, gender- and BMI-matched healthy controls. Second, steatosis (but not energetics) is independently associated with impaired diastolic strain rate. Third, reduced $\mathrm{VO}_{2}$ max is related to elevated MTG, and this relationship may be mediated through impaired diastolic strain rate.

While steatosis is known to be associated with diastolic dysfunction in diabetes [28], data on steatosis in $\mathrm{HFpEF}$ and its relationship with cardiac function and exercise capacity are lacking. There is only one study 


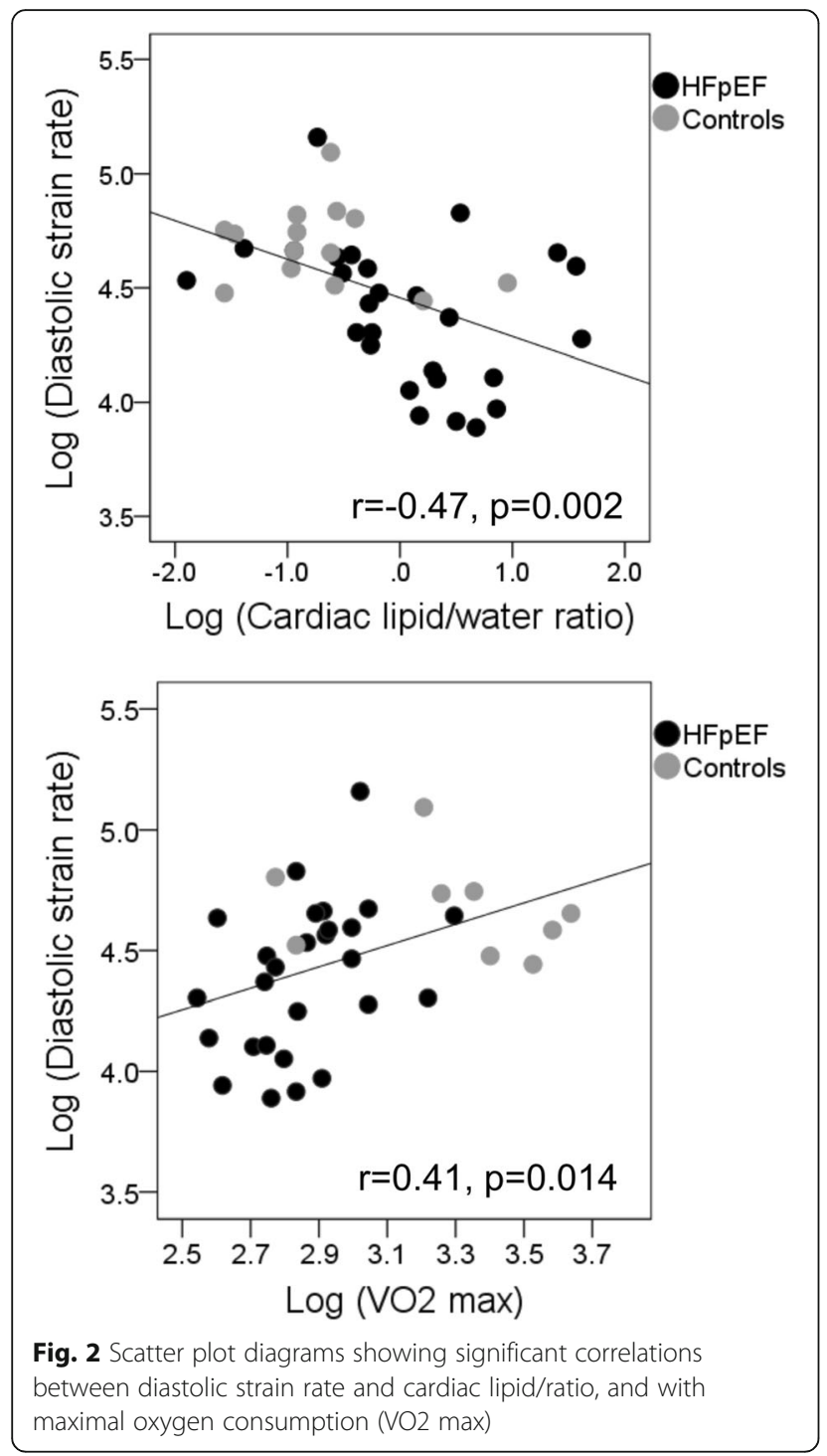

recently showing steatosis in a small number of young women $(n=5)$ with microvascular dysfunction and subclinical HFpEF [15]. Here, we demonstrate for the first time MTG in a larger number $(n=27)$ of typically elderly patients with HFpEF, who have limiting exertional dyspnoea, abnormal diastolic function demonstrated by Doppler echo and CMR FT, and objective evidence of exercise-limitation by CPET. We categorized our HFpEF cohort as mild as although they fulfilled the criteria based on the guidelines $[18,19]$ their diastolic abnormalities were mildly impaired which were likely due to the effect of taking diuretics.

Importantly, we show that elevated MTG independently correlated with impaired diastolic strain rate. The mechanisms leading to such pronounced steatosis remain to be completely understood, but it is well established that cardiac hypertrophy is associated with altered
Table 3 Bivariate correlations and multivariate correlations for diastolic strain rate and $\mathrm{VO}_{2}$ max

\begin{tabular}{|c|c|c|c|c|}
\hline & \multicolumn{2}{|c|}{ Bivariate } & \multicolumn{2}{|c|}{ Multivariate } \\
\hline & $\mathrm{R}$ & $P$-Value & $\bar{\beta}$ & $P$-Value \\
\hline \multicolumn{5}{|l|}{ Diastolic strain rate } \\
\hline Myocardial triglyceride & -0.47 & 0.002 & -0.58 & 0.001 \\
\hline PCr/ATP ratio & 0.41 & 0.026 & 0.11 & 0.45 \\
\hline Age & -0.18 & 0.26 & - & - \\
\hline LVM/EDV & -0.20 & 0.30 & - & - \\
\hline SBP & -0.16 & 0.92 & - & - \\
\hline BNP & -0.19 & 0.29 & - & - \\
\hline LA size & -0.10 & 0.59 & - & - \\
\hline \multicolumn{5}{|l|}{$\mathrm{VO}_{2} \max$} \\
\hline Myocardial triglyceride & -0.34 & 0.04 & -0.35 & 0.23 \\
\hline Diastolic strain rate & 0.41 & 0.014 & 0.57 & 0.007 \\
\hline PCr/ATP ratio & 0.49 & 0.014 & 0.35 & 0.13 \\
\hline Age & -0.29 & 0.08 & - & - \\
\hline LVM/EDV & -0.33 & 0.11 & - & - \\
\hline SBP & -0.20 & 0.28 & - & - \\
\hline BNP & 0.21 & 0.25 & - & - \\
\hline LA size & -0.21 & 0.28 & - & - \\
\hline
\end{tabular}

PCr Phosphocreatine, ATP Adenosine triphosphate, LVM Left ventricular mass, EDV End-diastolic volume, SBP Systolic blood pressure, BNP Brain natriuretic peptide, LA Left atrial

myocardial substrate metabolism with a shift towards glucose and away from FA oxidation, leading to increased myocardial lipid accumulation, non-oxidative metabolism and reduced cardiac function [8, 29, 30]. While our data show that MTG and diastolic strain rate have significant correlations with exercise capacity, only diastolic strain rate independently correlated with $\mathrm{VO}_{2}$ max. This is not surprising given the fact the abnormal resting diastolic function has been shown to be associated with worsening of exercise capacity [31]. Furthermore the underlying mechanism of MTG directly affecting $\mathrm{VO}_{2}$ max is unclear and could potentially be mediated through reduced diastolic strain rate. While our data do not prove a direct causal link, they may suggest a pathophysiological role of steatosis in the development of diastolic dysfunction and reduced functional capacity in mild HFpEF. It would have been interesting to demonstrate more profound impairment in diastolic function along with changes in metabolic substrate metabolism during exercise. In fact, Phan et al has previously shown more profound diastolic abnormalities during stress radionuclide ventriculography in HFpEF, which were not seen at rest [16]. In future, taking blood samples for plasma substrate and metabolomics during exercise to assess changes in substrate metabolism would support the findings in the present study. 
In the present study, our HFpEF patients had increased LV wall thickness and significant concentric remodeling, despite normal LV mass. Given our cross-sectional study design, we cannot determine if cardiac steatosis causes LV remodeling or vice versa. However, several studies using animal models of pathological hypertrophy have demonstrated a causal link between steatosis and development of cardiac remodeling $[29,30]$. Cardiac steatosis can lead to production of harmful intermediates and apoptosis. These can stimulate hypertrophic signalling leading to concentric LV hypertrophy followed by eventually a dilated phenotype [32, 33]. The question remains, what is the potential driving factor for metabolic alteration and steatosis in HFpEF? Kato et al recently showed that HFpEF patients have reduced coronary reserve due to microvascular dysfunction [34], and this has been proposed as a possible trigger of the metabolic switch and steatosis prior to the development of cardiac hypertrophy [15].

Impaired myocardial energetics has been demonstrated in asymptomatic diastolic dysfunction due to obesity [35] and also in HFpEF [16]. Here we extend the findings by showing significant correlations between energetics, diastolic strain rate and exercise capacity in mild HFpEF. Metabolic alterations in hypertrophied hearts have been shown to include impaired myocardial energetics [30], and detrimental effects of ceramides on mitochondria can lead to reduced intracellular ATP production, apoptosis and reduced cardiac function [36]. This may be a mechanism behind the observed relationship between myocardial energetics and function in the current study. Although both myocardial energetics status and triglyceride content correlated with diastolic strain rate, only steatosis independently correlated with diastolic strain rate. These are in keeping with a previous study in patients with type 2 diabetes mellitus and preserved LVEF, showing an independent association of steatosis with diastolic dysfunction [28]. This is an important finding and suggests that the pathophysiological cascade leading to diastolic dysfunction in HFpEF may involve steatosis at an earlier stage than energetic derangement - an observation with potential consequences in our search for therapeutic targets in HFpEF.

The current study provides novel insight into the pathophysiological role of steatosis in mild HFpEF. While inhibition of FA oxidation and stimulation of glucose oxidation may be beneficial in HF due to ischaemic insults [37, 38], cardiac lipid modulation by augmenting FA oxidation might be an alternative therapeutic strategy in non-ischaemic HF $[8,30]$. Since myocardial steatosis is modifiable, novel metabolic therapies aimed at improving/preserving cardiac function and exercise capacity, thus delaying the progression to the more severe form of HFpEF by reducing MTG should be tested. Potential therapeutic agents are glucagon-like peptide-1 receptor (GLP-1) agonists, mineralocorticoid receptor blockers such as eplerenone, and fenofibrate, which have been shown to reduce myocardial steatosis in Type 2 Diabetes [33, 39, 40].

\section{Study limitations}

As this study is limited by a small sample size, further corroboration of these findings in larger-scale multi-centre studies is required. Although the current study is powered to detect a difference, it may be underpowered to confirm the null hypothesis. Therefore, the non-statistical age difference between control and HFpEF cohorts would imply that age could still be a confounding variable for the difference in MTG observed. However, it would not be physiologically possible for a small age difference to result in > two-fold increase in MTG. In the present study, we excluded patients with a history of coronary artery disease and there was no evidence of myocardial infarction on CMR LGE imaging. Myocardial stress perfusion to assess microvascular dysfunction was not performed, as we did not want to burden these frail patients with a longer scan protocol. Thus, reduced coronary microvascular reserve during stress cannot be excluded as a potential mechanism contributing to the metabolic alteration in HFpEF. Due to the relatively long scan protocol, it was not feasible to perform reproducibility tests in the current study, thus reproducibility of lipid/water and PCr/ATP techniques in HFpEF is unknown. However, the reproducibility of these techniques have been shown to be excellent in healthy subjects $[11,20]$. The current study is not representative of the HFpEF population as patients with diabetes and uncontrolled hypertension were excluded. In the future, the analysis should be repeated in a larger sample size including patients with these comorbidities. As there is lack of accepted standard MTG normal threshold, the interpretation of the results may be limited as the findings are based on the normal MTG value at our institution. The relatively low MTG (0.64) in controls could reflect the healthiness of the cohort control that is not representative of normal aging. Future studies will be needed to investigate the true MTG in old sedentary individuals. Similarly, the participation of very healthy subjects cannot be excluded as a potential factor contributing to the supra normal PCr/ATP. Although results vary slightly between centres, the mean PCr/ATP value for healthy controls in the current study is consistent with the values reported previously in this age group $[16,41]$. Finally, the observational nature of our study precludes inferences of attribution. Further interventional research is needed to examine the change in MTG or PCr/ATP to confirm causal relationship between diastolic dysfunction/ $/ \mathrm{VO}_{2} \max$ and metabolic abnormalities. 


\section{Conclusions}

Mild HFpEF is characterized by pronounced myocardial steatosis, impaired myocardial energetics, impaired diastolic strain rate and reduced $\mathrm{VO}_{2}$ max. Reduced $\mathrm{VO}_{2}$ max may be attributable to elevated MTG via reducing diastolic function. MTG is a promising therapeutic target in HFpEF, thereby potentially improving exercise capacity, and, ultimately, outcomes in this difficult-to-treat patient population.

\section{Abbreviations}

ATP: Adenosine triphosphate; BMI: Body mass index; BNP: Brain natriuretic peptide; bSSFP: Balanced steady state free precession; CMR: Cardiovascular magnetic resonance; CMRS: Cardiovascular magnetic resonance spectroscopy; CPET: Cardiopulmonary exercise test; CSI: Chemical shift imaging; ECG: Electrocardiogram; Echo: Echocardiogram; EDV: End diastolic volume; EF: Ejection fraction; ESV: End systolic volume; FA: Fatty acid; FT: Feature tracking; HF: Heart failure; HFpEF: Heart failure with preserved ejection fraction; LA: Left atrium/left atrial; LGE: Late gadolinium enhancement; LV: Left ventricle/left ventricular; MTG: Myocardial triglyceride; PCr/ATP: Phosphocreatine-to-adenosine triphosphate; RER: Respiratory Exchange ratio; SBP: Systolic blood pressure; STEAM: Stimulated echo; TE: Echo time; TR: Repetition time; $\mathrm{VO}_{2}$ max: Maximal oxygen consumption

\section{Acknowledgements}

Not applicable.

\section{Funding}

This work was supported by Chest Heart and Stroke Association, Scotland. MM acknowledges support from the National University of Malaysia and Ministry of Higher Education Malaysia. SN and OR acknowledge support from the Oxford NIHR Biomedical Research Centre and the Oxford British Heart Foundation Centre of Research Excellence. JES is a Senior BHF Basic Science Research Fellow (FS/11/50/29038). CTR is funded by a Sir Henry Dale Fellowship from the Wellcome Trust and the Royal Society [098436/Z/12/Z/ B]. OR is a BHF Clinical Intermediate Research Fellow FS/16/70/32157.

\section{Availability of data and materials}

Dataset used in this study are available from the corresponding author on reasonable request.

\section{Authors' contributions}

MM made substantial contributions to conception and design, performed image acquisition, data collection, statistical analysis, interpretation and manuscript preparation. NP and CH performed patient identification, image acquisition, data collection, contributed to intellectual content. JR contributed to acquisition and analysis of the data and critically evaluated the manuscript. BR, SD, EL, RA, VF and RB contributed to data collection, interpretation and critically evaluated the manuscript. JS and CR contributed to data analysis, critically evaluated the manuscript and provided expert opinion on the interpretation of the spectroscopic data. JF performed image acquisition, data analysis and critically evaluated the manuscript. TK contributed to supervision of all aspects of data collection and critically evaluated the manuscript. MF, HA and SN contributed to the intellectual input of the study design, data interpretation and critically evaluated the manuscript. OR contributed to intellectual input of the study design, oversaw manuscript preparation, data interpretation and performed statistical analysis. All authors participated fully in preparation of the manuscript, and have provided approval for its submission.

\section{Ethics approval and consent to participate}

All study participants gave informed consent to participate in the study, which was approved by the National Research Ethics Services Committee South Central (reference number 11/SC/0009).

\section{Consent for publication}

Not applicable. Patients' identifiers have been removed from all images and data reported in this manuscript.

\section{Competing interests}

The authors declare that they have no competing interests.

\section{Publisher's Note}

Springer Nature remains neutral with regard to jurisdictional claims in published maps and institutional affiliations.

\section{Author details}

${ }^{1}$ Division of Cardiovascular Medicine, Radcliffe Department of Medicine University of Oxford Centre for Clinical Magnetic Resonance Research (OCMR), University of Oxford, John Radcliffe Hospital, Headley Way, Oxford OX3 9DU, UK. ${ }^{2}$ National University of Malaysia Medical Centre, Kuala Lumpur, Malaysia. ${ }^{3}$ Divisions of Experimental Therapeutics and Cardiovascular Medicine, Radcliffe Department of Medicine, Oxford, UK. ${ }^{4} 1$ st Department of Cardiology, Aristotle University, Thessaloniki, Greece. ${ }^{5}$ Department of Medicine, John Radcliffe Hospital, Oxford, UK. ${ }^{6}$ Wolfson Brain Imaging Centre, University of Cambridge, Cambridge, UK. ${ }^{7}$ Norwich Medical School, Bob Champion Research and Education Building, James Watson Road, University of East Anglia Norwich Research Park, Norwich NR4 7UQ, UK.

Received: 17 October 2017 Accepted: 27 November 2018

Published online: 24 December 2018

\section{References}

1. Lam CS, Donal E, Kraigher-Krainer E, Vasan RS. Epidemiology and clinical course of heart failure with preserved ejection fraction. Eur J Heart Fail. 2011;13:18-28.

2. Borlaug BA, Paulus WJ. Heart failure with preserved ejection fraction: pathophysiology, diagnosis, and treatment. Eur Heart J. 2011:32:670-9.

3. Ponikowski P, Voors AA, Anker SD, Bueno H, Cleland JG, Coats AJ, Falk V, Gonzalez-Juanatey JR, Harjola VP, Jankowska EA, Jessup M, Linde C, Nihoyannopoulos P, Parissis JT, Pieske B, Riley JP, Rosano GM, Ruilope LM, Ruschitzka F, Rutten FH, van der Meer P. 2016 ESC guidelines for the diagnosis and treatment of acute and chronic heart failure: the task force for the diagnosis and treatment of acute and chronic heart failure of the European Society of Cardiology (ESC)developed with the special contribution of the heart failure association (HFA) of the ESC. Eur Heart J. 2016:37:2129-200

4. Yousaf F, Collerton J, Kingston A, Kenny A, Davies K, Jagger C, Robinson L, Kirkwood TB, Keavney B. Prevalence of left ventricular dysfunction in a UK community sample of very old people: the Newcastle $85+$ study. Heart. 2012;98:1418-23.

5. Correa de Sa DD, Hodge DO, Slusser JP, Redfield MM, Simari RD, Burnett JC, Chen $\mathrm{HH}$. Progression of preclinical diastolic dysfunction to the development of symptoms. Heart. 2010;96:528-32.

6. Ammar KA, Jacobsen SJ, Mahoney DW, Kors JA, Redfield MM, Burnett JC Jr, Rodeheffer RJ. Prevalence and prognostic significance of heart failure stages: application of the American College of Cardiology/American Heart Association heart failure staging criteria in the community. Circulation. 2007; 115:1563-70.

7. Neubauer S. The failing heart--an engine out of fuel. N Engl J Med. 2007; 356:1140-51.

8. Abdurrachim D, Luiken JJ, Nicolay K, Glatz JF, Prompers JJ, Nabben M. Good and bad consequences of altered fatty acid metabolism in heart failure: evidence from mouse models. Cardiovasc Res. 2015;106:194-205.

9. Unger RH. Lipotoxic diseases. Annu Rev Med. 2002;53:319-36.

10. Goldberg IJ, Trent CM, Schulze PC. Lipid metabolism and toxicity in the heart. Cell Metab. 2012;15:805-12

11. Rial B, Robson MD, Neubauer S, Schneider JE. Rapid quantification of myocardial lipid content in humans using single breath-hold $1 \mathrm{H}$ MRS at 3 tesla. Magn Reson Med. 2011;66:619-24.

12. Levelt E, Mahmod M, Piechnik SK, Ariga R, Francis JM, Rodgers CT, Clarke WT, Sabharwal N, Schneider JE, Karamitsos TD, Clarke K, Rider OJ, Neubauer $\mathrm{S}$. Relationship between left ventricular structural and metabolic remodeling in type 2 diabetes. Diabetes. 2016;65:44-52.

13. Mahmod M, Bull S, Suttie JJ, Pal N, Holloway C, Dass S, Myerson SG, Schneider JE, De Silva R, Petrou M, Sayeed R, Westaby S, Clelland C, Francis JM, Ashrafian H, Karamitsos TD, Neubauer S. Myocardial steatosis and left ventricular contractile dysfunction in patients with severe aortic stenosis. Circ Cardiovasc Imaging. 2013;6:808-16. 
14. Barger PM, Brandt JM, Leone TC, Weinheimer CJ, Kelly DP. Deactivation of peroxisome proliferator-activated receptor-alpha during cardiac hypertrophic growth. J Clin Invest. 2000;105:1723-30.

15. Wei J, Nelson MD, Szczepaniak EW, Smith L, Mehta PK, Thomson LE, Berman DS, Li D, Bairey Merz CN, Szczepaniak LS. Myocardial steatosis as a possible mechanistic link between diastolic dysfunction and coronary microvascular dysfunction in women. Am J Physiol Heart Circ Physiol. 2016;310:H14-9.

16. Phan TT, Abozguia K, Nallur Shivu G, Mahadevan G, Ahmed I, Williams L, Dwivedi G, Patel K, Steendijk P, Ashrafian H, Henning A, Frenneaux M. Heart failure with preserved ejection fraction is characterized by dynamic impairment of active relaxation and contraction of the left ventricle on exercise and associated with myocardial energy deficiency. J Am Coll Cardiol. 2009:54:402-9.

17. Moody WE, Taylor RJ, Edwards NC, Chue CD, Umar F, Taylor TJ, Ferro CJ, Young AA, Townend JN, Leyva F, Steeds RP. Comparison of magnetic resonance feature tracking for systolic and diastolic strain and strain rate calculation with spatial modulation of magnetization imaging analysis. J Magn Reson Imaging. 2015;41:1000-12.

18. Yancy CW, Jessup M, Bozkurt B, Butler J, Casey DE Jr, Drazner MH, Fonarow GC, Geraci SA, Horwich T, Januzzi JL, Johnson MR, Kasper EK, Levy WC, Masoudi FA, McBride PE, McMurray JJ, Mitchell JE, Peterson PN, Riegel B, Sam F, Stevenson LW, Tang WH, Tsai EJ, Wilkoff BL. 2013 ACCF/AHA guideline for the management of heart failure: a report of the American College of Cardiology Foundation/American Heart Association task force on practice guidelines. J Am Coll Cardiol. 2013;62:e147-239.

19. Vasan RS, Levy D. Defining diastolic heart failure: a call for standardized diagnostic criteria. Circulation. 2000;101:2118-21.

20. Tyler DJ, Emmanuel Y, Cochlin LE, Hudsmith LE, Holloway CJ, Neubauer S, Clarke K, Robson MD. Reproducibility of 31P cardiac magnetic resonance spectroscopy at 3 T. NMR Biomed. 2009;22:405-13.

21. Robson MD, Tyler DJ, Neubauer S. Ultrashort TE chemical shift imaging (UTE-CSI). Magn Reson Med. 2005;53:267-74.

22. Dass S, Cochlin LE, Suttie JJ, Holloway CJ, Rider OJ, Carden L, Tyler DJ, Karamitsos TD, Clarke K, Neubauer S, Watkins H. Exacerbation of cardiac energetic impairment during exercise in hypertrophic cardiomyopathy: a potential mechanism for diastolic dysfunction. Eur Heart J. 2015;36:1547-54.

23. Hudsmith LE, Petersen SE, Francis JM, Robson MD, Neubauer S. Normal human left and right ventricular and left atrial dimensions using steady state free precession magnetic resonance imaging. J Cardiovasc Magn Reson. 2005:7:775-82.

24. Nagueh SF, Appleton CP, Gillebert TC, Marino PN, Oh JK, Smiseth OA, Waggoner AD, Flachskampf FA, Pellikka PA, Evangelista A.

Recommendations for the evaluation of left ventricular diastolic function by echocardiography. J Am Soc Echocardiogr. 2009;22:107-33.

25. Guazzi M, Myers J, Peberdy MA, Bensimhon D, Chase P, Arena R. Cardiopulmonary exercise testing variables reflect the degree of diastolic dysfunction in patients with heart failure-normal ejection fraction. J Cardiopulm Rehabil Prev. 2010;30:165-72.

26. Garcia EL, Menezes MG, Stefani Cde M, Danzmann LC, Torres MA. Ergospirometry and echocardiography in early stage of heart failure with preserved ejection fraction and in healthy individuals. Arq Bras Cardiol. 2015;105:248-55

27. Schneider J. Age dependency of oxygen uptake and related parameters in exercise testing: an expert opinion on reference values suitable for adults. Lung. 2013;191:449-58.

28. Rijzewijk L, van der Meer RW, Smit JW, Diamant M, Bax JJ, Hammer S, Romijn JA, de Roos A, Lamb HJ. Myocardial steatosis is an independent predictor of diastolic dysfunction in type 2 diabetes mellitus. J Am Coll Cardiol. 2008:52:1793-9.

29. Chiu HC, Kovacs A, Ford DA, Hsu FF, Garcia R, Herrero P, Saffitz JE, Schaffer JE. A novel mouse model of lipotoxic cardiomyopathy. J Clin Invest. 2001; 107:813-22.

30. Kolwicz SC Jr, Olson DP, Marney LC, Garcia-Menendez L, Synovec RE, Tian R. Cardiac-specific deletion of acetyl CoA carboxylase 2 prevents metabolic remodeling during pressure-overload hypertrophy. Circ Res. 2012;111:728-38.

31. Grewal J, McCully RB, Kane GC, Lam C, Pellikka PA. Left ventricular function and exercise capacity. JAMA. 2009;301:286-94.

32. Nelson MD, Victor RG, Szczepaniak EW, Simha V, Garg A, Szczepaniak LS. Cardiac steatosis and left ventricular hypertrophy in patients with generalized lipodystrophy as determined by magnetic resonance spectroscopy and imaging. Am J Cardiol. 2013;112:1019-24.
33. Ramirez E, Klett-Mingo M, Ares-Carrasco S, Picatoste B, Ferrarini A, Ruperez FJ, Caro-Vadillo A, Barbas C, Egido J, Tunon J, Lorenzo O. Eplerenone attenuated cardiac steatosis, apoptosis and diastolic dysfunction in experimental type-II diabetes. Cardiovasc Diabetol. 2013;12:172.

34. Kato S, Saito N, Kirigaya H, Gyotoku D, linuma N, Kusakawa Y, lguchi K, Nakachi T, Fukui K, Futaki M, Iwasawa T, Kimura K, Umemura S. Impairment of coronary flow reserve evaluated by phase contrast cine-magnetic resonance imaging in patients with heart failure with preserved ejection fraction. J Am Heart Assoc. 2016;5(2):e002649. Published online 2016 Feb 23. https://doi.org/10.1161/JAHA.115.002649.

35. Rider OJ, Francis JM, Ali MK, Holloway C, Pegg T, Robson MD, Tyler D, Byrne J, Clarke K, Neubauer S. Effects of catecholamine stress on diastolic function and myocardial energetics in obesity. Circulation. 2012;125:1511-9.

36. Parra V, Moraga F, Kuzmicic J, Lopez-Crisosto C, Troncoso R, Torrealba N, Criollo A, Diaz-Elizondo J, Rothermel BA, Quest AF, Lavandero S. Calcium and mitochondrial metabolism in ceramide-induced cardiomyocyte death. Biochim Biophys Acta. 2013;1832:1334-44.

37. Hafstad AD, Khalid AM, Hagve M, Lund T, Larsen TS, Severson DL, Clarke K, Berge RK, Aasum E. Cardiac peroxisome proliferator-activated receptor-alpha activation causes increased fatty acid oxidation, reducing efficiency and post-ischaemic functional loss. Cardiovasc Res. 2009;83:519-26.

38. Tian R, Abel ED. Responses of GLUT4-deficient hearts to ischemia underscore the importance of glycolysis. Circulation. 2001;103:2961-6.

39. Monji A, Mitsui T, Bando YK, Aoyama M, Shigeta T, Murohara T. Glucagonlike peptide-1 receptor activation reverses cardiac remodeling via normalizing cardiac steatosis and oxidative stress in type 2 diabetes. Am 」 Physiol Heart Circ Physiol. 2013;305:H295-304.

40. Forcheron F, Basset A, Abdallah P, Del Carmine P, Gadot N, Beylot M. Diabetic cardiomyopathy: effects of fenofibrate and metformin in an experimental model - the Zucker diabetic rat. Cardiovasc Diabetol. 2009;8:16.

41. Nathania M, Hollingsworth KG, Bates M, Eggett C, Trenell MI, Velicki L, Seferovic PM, MacGowan GA, Turnbull DM, Jakovljevic DG. Impact of age on the association between cardiac high-energy phosphate metabolism and cardiac power in women. Heart. 2018;104:111-8.

Ready to submit your research? Choose BMC and benefit from:

- fast, convenient online submission

- thorough peer review by experienced researchers in your field

- rapid publication on acceptance

- support for research data, including large and complex data types

- gold Open Access which fosters wider collaboration and increased citations

- maximum visibility for your research: over $100 \mathrm{M}$ website views per year

At $\mathrm{BMC}$, research is always in progress.

Learn more biomedcentral.com/submissions 